\title{
20. Zur Axiomatik der ganzen und der reellen Zahlen.
}

\author{
Von Teiji TAKAGI, M. I. A.
}

(Comm. March 12, 1945.)

\section{Ganze Zahlen.}

Das System der ganzen Zahlen laesst sich auf Grund der folgenden Axiome einfuehren. Indem wir uns in dieser summarischen Mitteilung Einfachheitshalber der Sprache der Mengenlehre (Klassen-lehre) bedienen, bezeichnen wir mit $N$ die Menge der ganzen Zahlen.

Axiom 1. $N$ lasst sich eine (nicht identische) ein-eindeutige Abbildung in sich zu: $x \rightleftarrows \varphi(x)$, wobei $x$ sowie $\varphi(x)$ die saemtlichen Elemente von $N$ durchlaufen.

Wir'schreiben $x^{+}$bez. $x^{-}$für $\varphi(x)$ bez. $\phi^{-1}(x)$, und wenn $M \subset N, M^{ \pm}$für die Menge $\left\{x^{ \pm} ; x \in M\right\}$, also beispielsweise $N=N^{+}=N^{-}$.

Axion 2. $N$ ist minimum ihrer Art oder irreduzibel, d.h. wernn $M \subset N$ und $M=M^{+}$(folglich auch $M=M^{-}$) so fällt $M$ mit $N$ zusammen. (Prinzip der mathematischen Induktion.)

Zusatz. Für jedes Element von $N$ gilt $a \neq a^{+}$. Wäre $a=a^{+}$, so gelte für $M=N-a<N, M=M{ }^{+}$in Widerpruch mit Axiome 2.

Eine Untermenge $M$ von $N$, die mit $x$ zugleich auch $x^{+}$(bez. $x^{-}$), enthält, heisse eine Progression (bez. Regression).

Satz. 1. Vereinigung und Durchschnitt von Progressionen sind wieder Progressionen; mit $M$ sind $M^{ \pm}$Progressionen. Ebenso für die Regressionen.

Die Durchschnitt aller Progressionen, bez. Regressionen, die das Element $a$ enthalten, sei mit $K(a)$ bez. $L(a)$ bezeichnet.

Satz 2. $K(a)^{ \pm}=K\left(a^{ \pm}\right) . \quad L(a)^{ \pm}=L\left(a^{ \pm}\right)$.

$K(a)^{+}$ist eine Progression, die $a^{+}$enthält, folglich $\supset K\left(a^{+}\right) \cdot K\left(a^{+}\right)^{-}$ ist eine Progression, die $a$ enthält, folglich $\supset K(a)$. Daher $K\left(a^{+}\right) \supset K(a)^{+}$. Also $K(a)^{+}=K\left(a^{+}\right)$. Ebenso für $K\left(a^{-}\right)$. Dual $\left(\varphi^{-1} \operatorname{statt}(\varphi)\right)$ für $L(a)$.

Satz 3. $K(a)=\vee\left(a, K\left(a^{+}\right)\right), L(a)=\vee\left(a, L\left(a^{-}\right)\right)$.

Die rechte Seite ist eine Progression, diè $a$ enthält, folglich $\supset K(a)$. Anderseits ist $K(a)-a$ eine Progression, die $a^{+}$enthält, folglich $\supset K\left(a^{+}\right)$, daher $K(a) \supset \vee\left(a, K\left(a^{+}\right)\right)$. Also $K(a)=\vee\left(a, K\left(a^{+}\right)\right)$. Dual fǘr $L(a)$.

Satz 4. $N=\vee\left(K(a), L\left(a^{-}\right)\right)$.

$M$ sei die rechte Seite. Dann ist $M^{+}==\vee\left(K(a)^{+}, L\left(a^{-}\right)^{+}\right)=\vee\left(K\left(a^{+}\right)\right.$, $L(a))=\vee\left(K\left(a^{+}\right), a, L\left(a^{-}\right)\right)=\vee\left(K(a), L\left(a^{-}\right)\right)=M . \quad$ Folglich $M=N$. 
Nunmehr sind zwei Fälle zu unterscheiden :

$1^{\circ} \quad$ (Zyklus) Jede Progression fällt mit $N$ zusammen.

$2^{\circ}$ (Zweiseitig unendliche Reihe) Es gibt eine Progression, echten Teil von $N$.

Folgendes ist zu bemerken: Wenn für ein Element $a, N=K(a)$, dann gilt für jedes Element $x, N=K(x)$. Jede Progression deckt $N . \quad N$ ist ein Zyklus. - Denn aus $N=K(x)$ folgt $N=N^{ \pm}=K(x)^{ \pm}=K\left(x^{ \pm}\right)$. Fur die nicht leere Menge $M=\{x ; K(x)=N\}$ gilt somit $M=M^{+}$, also $M=N$, vermöge Axiome 2.

Wir beschäftigen uns zunächst mit Fall $2^{\circ}$, in welchem die Sätze 5-9 gelten.

Satz 5. $a^{-} \notin K(a), a^{+} \notin L(a)$.

Aus $a^{-} \in K(a)$ folgte $a \in K(a)^{+}, K(a)=\vee\left(a, K(a)^{+}\right)=K(a)^{+}, K(a)$ $=N$.

Satz 6. Mit $x \in K(a)$ ist $x^{-} \in K(a)$, ausser wenn $x=a$. Mit $x \in L(a)$ ist $x^{+} \in L(a)$, ausser wenn $x=a$.

Aus $x \in K(a)$ folgt $x^{-} \in K\left(a^{-}\right)=\vee\left(a^{-}, K(a)\right)$, woraus mit $x \neq a, x^{-} \neq$ $a^{-}, x^{-} \in K(a)$. Dual für $L(a)$.

Satz 7. $K(a)$ und $L\left(a^{-}\right)$sind komplementär in Bezug auf $N$.

Denn sei etwa $d \in D=\wedge\left(K(a), L\left(a^{-}\right)\right)$. Dann wäre $d \neq a, d \in K(a)$, daher $d^{-} \in K(a)$, somit $d^{-} \in D$. Ebenso $d \neq a^{-}, d \in L\left(a^{-}\right)$, daher $d^{+} \in L\left(a^{-}\right)$, $d^{+} \in D$. Somit $D^{+}=D=N$, in Widerspruch mit $a \notin D$.

Satz 8. Wenn $a \neq b$, so ist $K(a)<K(b)$ oder $K(a)>K(b)$, jenachdem $a$ zu $\mathrm{K}(b)$ gehört oder nicht.

Aus $a \in K(b)$ folgt $K(a) \subset K(b)$. Wäre nun $K(b)=K(a)=\vee(a$, $\left.K(a)^{+}\right)$, dann wäre, weil $a \neq b, b \in K(a)^{-}<K(a), K(b)<K(a)$, was ausgeschlossen ist. Daler $K(a)<K(b)$. Wenn $a \notin K(b)$, so ist $a \in L\left(b^{-}\right)$, folglich $L(a) \subset L\left(b^{-}\right)$, und indem man zum Komprlementäre tibergeht, $K\left(a^{+}\right)$ $>K(b), K(a)>K(b)$.

Wir setzen $a>b$ bez. $a<b$, jenachdem $K(a)<K(b)$ oder $K(a)>K(b)$.

Satz. 9. Aus $a>b, b>c$ folgt $a>c$.

Ein beliebiges Element von $N$ setzen wir $=0,0^{+}=1,0^{-}=-1$, u.s.w. Die Elemente von $K(1)$ bez. $L(-1)$ seien die positiven bez. die negativeu ganzen Zahlen. Alle $K(a)$ sowie alle $L(a)$ sind isomorph; $K(a)$ und $L(b)$ dual reziprok, $K(1)$ ist mit dem Peano'schen natürlichen Zahlensysteme isomorph; u.s. w., u. s. w.

Die Addition der ganzen Zahlen lässt sich auf Grund von Satz 8 nach Dede- 
kind'schen Diagonalverfahren,") oder in direktem Anschluss an die Axiome nach Herrn Kalmars Konstruktion ${ }^{2)}$ definiren, was wir nicht weiter ausführen wollen.

Es bleibt den anderen Falle, den Zyklus, zu betrachten tibrig. Da wir nummehr die ganzen Zahlen voraussetzen dürfen, verwenden wir dieselben zum Numeriren der Elemente von $N$. Wir beginnen mit einem beliebigen Elemente von $N$, das wir mit 1 bezeichnen und fahren mit den Elementen von $K(1)$ so weiter fort, dass $\varphi(a)$ die Nummer $a+1$ erhält. $N=K(1)$ enthält aber das Element $1^{-}$. Hat es die Nummer $n$, so sind mit 1, 2, ., $n$ alle Elemente von $N$ erschöpft vermöge des Axiomes 2. Es ist $N$ eine endliche Menge. In Bild gesprochen, ist eine endliche Menge diejenige, deren sämtlichen Elemente in einen Kreis arrangiren lassen.

\section{Reelle Zahlen.}

$N$ sei die Menge der reellen Zahlen.

Axiom 1. $N$ ist ein Linearkontinuum, d.h. $N$ ist geordnet, im Dedekind'schen Sinne stetig, und beiderseits offen.

Axiom 2. $\quad N$ ist minimum ihrer Art in dem Sinne, dass jedes Linearkontinuum, Untermenge von $N$, mit $N$ ähnlich in Bezug auf die Ordnung in $N$ ist.

Auf Grund von Axom 1 nehmen wir in $N$ eine beiderseits unendliche Reihe an, deren Elemente wir ordnungsgemäss mit den ganzen Zahlen bezeichnen. Sodann nehmen wir in jedem Intervall $(n, n+1)$ ein Element, das wir mit $(2 n+1) / 2$ bezeichnen. Indem wir auf diese Weise unbegrenzt fortfahren, erhalten wir eine in sich dichte Menge $M$. Jede wachsende Folge in $M$ hat eine obere Grenze in $N$. Ftigt man diese zu $M$ hinzu, so erhalten wir ein Linearkontinuum $M$ in $N$. Da nach Axiom 2, $M$ mit $N$ ähnlich ist, so können wir die Bezeichnung der Elemente von $M$ auf die entsprechenden von $N$ uibertragen und erhalten für die Elemente von $N$ eine Darstellung in der Form der dyadischen (unendlichen) Brtichen. Hiermit wollen wir unseres Axiomensystem gerechtfertigt haben!

1) Was sind und was sollen die Zahlen, $\$ 9$.

2) Vgl. T. Takagi, Diese Proc., 7, 29 (1931). 